\title{
Balancing of Agricultural Census Data by using Discrete Optimization*
}

\author{
Bianchi Gianpiero $^{1}$ - Bruni Renato ${ }^{2} \cdot$ Reale Alessandra $^{3}$
}

\begin{abstract}
In the case of large-scale surveys, such as a Census, data may contain errors or missing values. An automatic error correction procedure is therefore needed. We focus on the problem of restoring the consistency of agricultural data concerning cultivation areas and number of livestock, and we propose here an approach to this balancing problem based on Optimization. Possible alternative models, either linear, quadratic or mixed integer, are presented. The mixed integer linear one has been preferred and used for the treatment of possibly unbalanced data records. Results on real-world Agricultural Census data show the effectiveness of the proposed approach.
\end{abstract}

Keywords Data Mining, Balancing Problems, Information Reconstruction, Mixed Integer Linear Models

\section{Introduction}

A Census of Agriculture is a very complex, important and expensive operation for a National Statistic Office. It is an essential activity, periodically performed for monitoring the agricultural sector (see also [14]). Data collected in such a process have therefore a great intrinsic economic value, and moreover, in the case of EU countries, constitute a basis for assigning financial resources, planning production, and for several other economical European policies. As in any other large-scale survey, however, those data may contain errors or missing values, due to a variety of reasons. Nonetheless, correct information must be published and provided to the EU level, also considering that large financial resources are allocated to the sector. Therefore, error detection and correction become crucial tasks. This kind of activity is generally called Information Reconstruction, or also Data Cleaning, within the field of Data Mining (see also [18,23]), or Data Editing and Imputation within the field of Statistics (see also [12,31]). Note that, in contexts different from the Census, the possibility of reconstructing exact values could be useful also for counteracting possible opportunistic behaviors (e.g. willingly erroneous declarations), and knowing that exact values can be reconstructed could indeed prevent such opportunistic behaviors.

Data are generally organized into conceptual units called records (see also [28]). In the case of a Census of Agriculture, data are typically constituted by farm codes, cultivation codes, size of cultivation areas and other amounts, years, etc., so we restrict our attention to numerical data. Agriculture is a rich source of large data mining problems, and a recent overview on the use of data mining techniques in this field is in [25]. The above Information Reconstruction tasks, in particular, can be performed by following different approaches, each of which having its own features. A main approach is based on the use of rules, called edits, that each data record must respect in order to be declared exact (see e.g. [3,24]). Records not respecting such rules are declared erroneous. A seminal paper on the subject is [15]. However, satisfactory rules accuracy and computational efficiency often appear to be at odds. For this reason, rules are often converted into mathematical expressions, e.g. inequalities (see also [11]), and finding within a record the most probably erroneous fields or the most suitable values correcting those fields become nontrivial optimization problems (see e.g. [17] for an introduction to computational complexity). This allows to overcome the computational limits of other techniques (see e.g. [4,24,31]). Such a

\footnotetext{
* Work developed during the biennial research collaboration between the Italian Statistic Office (Istat) and the University of Roma "Sapienza" on the data processing of the 2010 Census of Italian Agriculture.

${ }^{1}$ Bianchi Gianpiero Istat, Dip. per i Censimenti e gli Archivi Amm. e Statistici (DICA)

Viale Oceano Pacifico 171, 00144 Roma, Italy

${ }^{2}$ Bruni Renato Università di Roma "Sapienza" Dip. di Ingegneria Informatica, Automatica e Gestionale (DIAG) Via Ariosto 25, 00185 Roma, Italy

e-mail: bruni@dis.uniroma1.it

${ }^{3}$ Reale Alessandra Istat, Dip. per i Censimenti e gli Archivi Amm. e Statistici (DICA)

Viale Oceano Pacifico 171, 00144 Roma, Italy
} 
methodology has been adopted within the data Editing and Imputation software system DIESIS $[9,10]$ and in other works such as $[12,27]$.

In the described Census, each farm specifies the cultivation area used for each cultivation and number of livestock for each type of animal, divided in some cases also by year. Moreover, they specify total areas and total numbers of livestock. However, those totals may be inconsistent with the mentioned detailed information, and a classical problem is restoring data consistency by correcting errors. These errors should be corrected by mathematically "guessing" the correct values, since it is clearly impossible to contact again the farm or inspect it somehow. The main issue is doing this on large data sets both efficiently and in order to obtain corrected data as similar as possible to the exact (but unknown) data. This work presents an innovative procedure for solving this problem based on optimization. In particular, Section 2 describes in detail the specific problem structure, analyzing also its connections to similar problems, and explains the development of the proposed integer linear programming model. Section 3 reports computational results in the case of the Italian Census of Agriculture 2010 ("Censimento Generale dell'Agricoltura 2010"), both for plants cultivations and for livestock. Note that, to the best of our knowledge, no previous attempt to treat this large-size Census problem with a discrete optimization approach was made, and only ad hoc procedures, designed by experts after an analysis of the specific available data, were used.

\section{Problem Structure and Optimization Model}

Data obtained from each farm during the described Census contain information (called microdata, information about details) about the cultivation area used by that farm for each cultivation and the number of livestock for each type of animal. Those data may sometimes be erroneous or missing, due to a variety of reasons. In such cases, errors should be automatically detected and corrected, i.e. the information that was corrupted and lost should be "reconstructed" in order to be as similar as possible to the unknown exact value. Moreover, each farm also declares other information (called macrodata, information about totals): the total cultivation area and the total number of livestock, and in some cases those totals are also divided into subtotals by year of planting. Clearly, balancing conditions must hold between all the above microdata and the corresponding macrodata: each total (or year subtotal) must be equal to the sum of those details concerning its parts. When such conditions do not hold, data are inconsistent.

Records incurring in this problem are detected by checking the balancing conditions, which are called balance edits. However, when a balance edit is violated, the error could be either on the detail side or on the total side of the equation. The less reliable information should be changed in order to restore consistency. It is generally assumed, in these cases, that details constitute the less reliable information, since totals have already been confirmed from other sources. This mathematical problem of adjusting the entries (here the microdata) of a large matrix to satisfy prior consistency requirements (here given by the macrodata) is called matrix balancing [29] and occurs in several fields, such as economics, urban planning, statistics, demography, etc. The problem is also related to the matrix rounding problem [1], consisting in rounding off the elements of a matrix consistently with its row and column sums, often arising in economic statistics, and belongs to the broad category of matrix scaling problems [2].

In some cases of matrix balancing problems the only aim is restoring balancing without further objectives, and iterative scaling algorithms can be used, e.g. the RAS algorithm [22]. In other cases, on the contrary, the variations introduced for balancing the matrix should pursue an objective that typically depends on the specific application. In the case of Census data, the choice of the objective is a delicate issue for avoiding data distortions, and makes this problem different from other types of balancing problems. Errors in microdata could broadly be divided into systematic errors and random errors [16]. Systematic errors are those caused by specific (and often traceable) mechanisms, e.g. usage of a wrong unit of measurement, OCR error, etc., and are generally treated during a preliminary correction phase [13]. Our central problem is therefore correcting microdata values affected by random errors. In this case, changes from the available microdata values should be minimized, according to specific distance criteria, since it is generally deemed that this should produce data as similar as possible to the unknown exact data (FellegiHolt paradigm $[15,24])$. An optimization approach is therefore required.

The models proposed for the above problem will be hereinafter explained by referring to the specific case of vineyards. This is one of the most important cases: dozens of grapes varieties exist, and they determine type and quality of wines produced. The case has great economic relevance and, due to its large dimension, is also computationally demanding. Moreover, those 
data are used when allocating European financial resources and when reorganizing wine production. However, the proposed models are clearly not limited to that case, but can be used for any other similar problem.

Each farm could have several vine types, and each of them could have been planted in a different time period (e.g. a specific year). Denote by

$I=\{1, \ldots, n\}$ the set of indices of all possible vine types; with $n=442$

$K=\{1, \ldots, m\}$ the set of indices of all possible time periods; with $m=6$.

For each farm, denote by

$a_{\mathrm{ik}}$ (real valued $\geq 0$ ) the area of vine type $i$ planted in period $k$ declared by the farm, with $i \in I$ and $k \in K$;

$a_{\mathrm{i} 0}$ (real valued $\geq 0$ ) the total area of vine type $i$ (planted during any of the periods) declared by the farm, with $i \in I$;

$T_{\mathrm{k}}$ (real valued $\geq 0$ ) the total vine area planted in period $k$ declared by the farm, with $k \in K$;

$T$ (real valued $\geq 0$ ) the total vine area owned by the farm.

In order to reconstruct the erroneous information, we need the following set of decision variables:

$x_{\mathrm{ik}}$ (real valued $\left.\geq 0, \leq S\right)=$ the area of vine type $i$ that, according to our reconstruction, has been planted in period $k$ by the farm, with $i \in I$ and $k \in K$.

$x_{\mathrm{i} 0}$ (real valued $\left.\geq 0, \leq m S\right)=$ the total area of vine type $i$ that, according to our reconstruction, has been planted (during any of the periods) by the farm, with $i \in I$.

In other words, $x_{\mathrm{ik}}$ is the correct value for $a_{\mathrm{ik}}$. When reconstructing information for a Census, as in the case of other large-scaled surveys, it is generally assumed that the changes introduced in the data should be somehow minimized. This because, in absence of further information, being as similar as possible to the exact (unknown) data corresponds to being as similar as possible to the available (even if possibly erroneous) data. By following this minimum change paradigm, two basic alternatives exist: one is minimizing the number of changes, the other minimizing the amount of those changes.

If we need to distinguish when our reconstruction provides a result which is different form the available declaration (i.e. a change), we need the following set of binary variables:

$$
y_{\mathrm{ik}}=\left\{\begin{array}{ll}
1 & \text { if } x_{\mathrm{ik}} \text { is different from } a_{\mathrm{ik}} \\
0 & \text { otherwise }
\end{array} \quad \forall i=1, \ldots, n \quad \forall k=0, \ldots, m\right.
$$

The presence of binary variables clearly has its impact on the complexity of the model: by adding the other constraints needed for this problem, which are linear, we obtain an Integer Linear Program. Minimizing the total number of changes corresponds to the following objective function

$$
\min \sum_{i=1}^{n} \sum_{k=0}^{m} y_{\mathrm{ik}}
$$

When variables $y$ are used, they should be linked to the $x$ variables by constraints imposing that $y_{\mathrm{ik}}$ takes value 1 when $x_{\mathrm{ik}}<>a_{\mathrm{ik}}$ (using a certain numerical precision), otherwise those variables could be inconsistent. There is no need for constraints imposing $y_{\mathrm{ik}}=0$ when $x_{\mathrm{ik}}=a_{\mathrm{ik}}$ because the objective (1) itself does that. Value $M$ is a real number greater than all possible values of the lefthand side of the following inequalities.

$$
\begin{array}{lll}
a_{\mathrm{ik}}-x_{\mathrm{ik}} \leq M y_{\mathrm{ik}} & \forall i=1, \ldots, n & \forall k=0, \ldots, m \\
x_{\mathrm{ik}}-a_{\mathrm{ik}} \leq M y_{\mathrm{ik}} & \forall i=1, \ldots, n & \forall k=0, \ldots, m
\end{array}
$$

When, on the other hand, we are interested in measuring the difference between our reconstruction $x_{\mathrm{ik}}$ and the available declaration $a_{\mathrm{ik}}$, we should consider a generic norm of this difference:

$$
\left\|a_{\mathrm{ik}}-x_{\mathrm{ik}}\right\|_{\mathrm{p}}
$$


Several norm types and norm-induced functions exist [5]. We consider more suitable to our reconstruction problems the following three:

- The squared Euclidean norm, defined as $\left(\|u-v\|_{2}\right)^{2}=\sum_{h=1}^{q}\left(u_{\mathrm{h}}-v_{\mathrm{h}}\right)^{2}$

- the so-called Manhattan norm, defined as $\|u-v\|_{1}=\sum_{h=1}^{q}\left|u_{\mathrm{h}}-v_{\mathrm{h}}\right|$

- the so-called Chebyshev norm, defined as $\|u-v\|_{\infty}=\max _{\mathrm{h}}\left\{\left|u_{\mathrm{h}}-v_{\mathrm{h}}\right|\right\}$.

Clearly, the structure of the optimization model that we must solve depends now on this choice. In the first case (squared Euclidean norm), minimizing the total amount of the changes corresponds to the following objective function, containing quadratic terms.

$$
\min \sum_{i=1}^{n} \sum_{k=0}^{m}\left(a_{\mathrm{ik}}-x_{\mathrm{ik}}\right)^{2}=\min \sum_{i=1}^{n} \sum_{k=0}^{m}\left(a_{\mathrm{ik}}^{2}-2 a_{\mathrm{ik}} x_{\mathrm{ik}}+x_{\mathrm{ik}}{ }^{2}\right)
$$

However, all of them are simply squared variables $\left(x_{\mathrm{ik}}\right)^{2}$, so they are strictly convex, and a conic combination of those strictly convex terms produces a separable strictly convex function [8]. By adding to that the linear terms of (4) and the constraints needed for this problem (described later), which are linear, the problem remains efficiently solvable (see e.g. [6,19]).

In the second case (Manhattan norm), there are absolute values in the objective. However, they can be easily linearized by introducing additional variables:

$$
s_{\mathrm{ik}}(\text { real valued } \geq 0)=\text { the value of }\left|a_{\mathrm{ik}}-x_{\mathrm{ik}}\right|, \quad \forall i=1, \ldots, n \quad \forall k=0, \ldots, m
$$

and linear constraints enforcing their meaning

$$
s_{\mathrm{ik}} \geq a_{\mathrm{ik}}-x_{\mathrm{ik}}, \quad s_{\mathrm{ik}} \geq x_{\mathrm{ik}}-a_{\mathrm{ik}} \quad \forall i=1, \ldots, n \quad \forall k=0, \ldots, m
$$

We can now minimize the linear function $\sum_{i=1}^{n} \sum_{k=0}^{m} s_{\text {ik }}$. When adding the other constraints needed for this problem, which are linear, the problem becomes an easily solvable Linear Program.

In the third case (Chebyshev norm), we have a min-max objective in the problem that again can be easily linearized by introducing one additional variable

$t($ real valued $\geq 0)=$ the value of $\max _{\mathrm{ik}}\left\{\left|a_{\mathrm{ik}}-x_{\mathrm{ik}}\right|\right\}, \quad \forall i=1, \ldots, n \quad \forall k=0, \ldots, m$

and linear constraints enforcing the above meaning

$$
t \geq a_{\mathrm{ik}}-x_{\mathrm{ik}}, \quad t \geq x_{\mathrm{ik}}-a_{\mathrm{ik}} \quad \forall i=1, \ldots, n \quad \forall k=0, \ldots, m
$$

We now simply minimize $t$. When adding the other constraints needed for this problem, which are linear, the problem becomes again an easily solvable Linear Program.

Clearly, also a combination of the above alternatives can be considered. The characteristics of the specific real problem will determine, from case to case, the choice of the objective among the described ones or their possible combinations. In our case, we consider more representative of the real problem's aim the minimization of the total number of changes, and, in second place, the minimization of the amount of those changes. This because a change with respect to a value that has been deliberately declared has intrinsically a very high cost. Therefore, we prefer maintaining the maximum number of those declared values, even if this may result in a greater amount of the changes that we are forced to introduce. The objective function becomes:

$$
\min \left(M^{\prime} \sum_{i=1}^{n} \sum_{k=0}^{m} y_{\mathrm{ik}}+\sum_{i=1}^{n} \sum_{k=0}^{m} s_{\mathrm{ik}}\right)
$$

where the first sums are multiplied by a numerical value $M^{\prime}$ 'weighting the relative importance of the first part with respect to the second one. We chose $M^{\prime}=S$, so that a single change weights as much as the maximum amount of a change. 
We now describe the balancing conditions that should be respected in our case. The sum of vine areas of any type planted in period $k$ must be equal to the total vine area planted in period $k$ (called balancing over vine types)

$$
\sum_{i=1}^{n} x_{\mathrm{ik}}=T_{\mathrm{k}} \quad \forall k \in K
$$

The sum of the areas of vine type $i$ planted in periods from 1 to $m$ must be equal to the area of the same vine type planted along all the periods (called balancing over time periods)

$$
x_{\mathrm{i} 0}=\sum_{k=1}^{m} x_{\mathrm{ik}} \quad \forall i \in I
$$

The sum of vine areas of any type planted in any period must be equal to the total vine area owned by the farm (called overall balancing)

$$
\sum_{i=1}^{n} \sum_{k=1}^{m} x_{\mathrm{ik}}=T
$$

Clearly, any other type of balancing condition could be expressed as other linear constraints. Note that the structure of balancing constraints (8) and (9) could be considered as defining a transportation problem (see e.g. [5,30]) with a set of origins $I$ and a set of destinations $K$, values $a_{\mathrm{i} 0}$ being the supply at origin $i$, values $T_{\mathrm{k}}$ being the demand at destination $k$, variables $x_{\mathrm{ik}}$ being the amount to be shipped from source $i$ to destination $k$. However, the values $a_{\mathrm{i} 0}$ are in our case declared values that we may change (using the $x_{\mathrm{i} 0}$ variables), and moreover there is no guarantee that the following condition, essential for the feasibility of a transportation problem, is respected:

$$
\sum_{i=1}^{n} a_{\mathrm{i} 0}=\sum_{k=1}^{m} T_{\mathrm{k}}
$$

The complete mixed integer linear programming model is therefore the following:

$$
\left\{\begin{array}{ccc}
\left.\min _{(M}, \sum_{i=1}^{n} \sum_{k=0}^{m} y_{\mathrm{ik}}+\sum_{i=1}^{n} \sum_{k=0}^{m} s_{\mathrm{ik}}\right) \\
\sum_{i=1}^{n} x_{\mathrm{ik}}=T_{\mathrm{k}} & \forall k \in K & \\
x_{\mathrm{i} 0}=\sum_{k=2}^{m} x_{\mathrm{ik}} & \forall i \in I & \\
\sum_{i=1}^{n} \sum_{k=1}^{m} x_{\mathrm{ik}}=T & \\
a_{\mathrm{ik}}-x_{\mathrm{ik}} \leq M y_{\mathrm{ik}} & \forall i=1, \ldots, n & \forall k=0, \ldots, m \\
x_{\mathrm{ik}}-a_{\mathrm{ik}} \leq M y_{\mathrm{ik}} & \forall i=1, \ldots, n & \forall k=0, \ldots, m \\
s_{\mathrm{ik}} \geq a_{\mathrm{ik}}-x_{\mathrm{ik}} & \forall i=1, \ldots, n & \forall k=0, \ldots, m \\
s_{\mathrm{ik}} \geq x_{\mathrm{ik}}-a_{\mathrm{ik}} & \forall i=1, \ldots, n & \forall k=0, \ldots, m \\
0 \leq x_{\mathrm{ik}} \leq S & \forall i \in I & \forall \\
0 \leq x_{\mathrm{i} 0} \leq m S \quad & \forall i \in I & \\
s_{\mathrm{ik}} \geq 0 & \forall i=1, \ldots, n & \forall k=0, \ldots, m \\
x_{\mathrm{ik}}, s_{\mathrm{ik}} \in \mathfrak{R} & \forall i=1, \ldots, n & \forall k=0, \ldots, m \\
y_{\mathrm{ik}} \in\{0,1\} & \forall i=1, \ldots, n & \forall k=0, \ldots, m
\end{array}\right.
$$




\section{Computational Analysis}

By sequentially solving the above model for each farm, we perform the requested Information Reconstruction process. This procedure was implemented in $\mathrm{C}++$, using ILOG Concert Technology [20] in order to express the described optimization models. The models themselves are solved by means of the state-of-the-art branch-and-cut (see e.g. [5,26]) procedure implemented by the solver ILOG Cplex [21], running on a 16 cores server having $128 \mathrm{~Gb}$ of RAM and Linux Operating System. The resulting software system has been tested for the treatment of data from the Italian Census of Agriculture 2010 (“Censimento Generale dell'Agricoltura 2010"), with specific respect to the cases of:

(I) Vineyards suitable for "controlled origin" wine, considered in Table 1;

(II) Vineyards not suitable for "controlled origin" wine, considered in Table 2;

(III) Generic cultivations, considered in Table 3;

(IV) Livestock, considered in Table 4.

Note that, in the last two cases, microdata are not subdivided by year of planting but by geographical area. In the above four cases, we report results for each Italian region and for all Italy ( $1^{\text {st }}$ column): the total number of farms not respecting the balancing conditions ( $2^{\text {nd }}$ column); the total number of records involved in those unsatisfied balancing conditions ( $3^{\text {rd }}$ column); the total number of changes operated by the reconstruction process $\left(4^{\text {th }}\right.$ column $)$. Moreover, we analyze in greater detail those changes: we report the percentages of area (or heads) modified by the procedure, computed with respect to the total area involved in that case (or to the total number of animals). Such modifications can be done by adding $\left(5^{\text {th }}\right.$ column $)$ and/or by subtracting $\left(6^{\text {th }}\right.$ column), and note that those quantities are not bounded to be equal, since errors are not so. Finally, we report the total processing time in seconds ( $7^{\text {th }}$ column).

The practical behavior of the proposed procedure should now be evaluated both from the computational and from the data quality points of view. As observable, the procedure is very fast: each single model is solved to optimality in extremely short times (generally about 0.02 sec.) so that the processing of all the Italian farms requires, for the 4 cases together, only about 50 minutes. The quality of the obtained data has been evaluated by considering: (i) the ability to restore balancing; and (ii) the variation produced in the data by the reconstruction process.

\begin{tabular}{|l|r|r|r|r|r|r|}
\hline \multicolumn{1}{|c|}{ Region } & Farms & \multicolumn{1}{c|}{ \# Records } & \multicolumn{1}{c|}{ Changes } & Added Area & $\begin{array}{c}\text { Subtracted } \\
\text { Area }\end{array}$ & Time (sec.) \\
\hline Piemonte & 696 & 2055 & 1866 & $0.15 \%$ & $-1.70 \%$ & 23.5 \\
\hline Valle d'Aosta & 22 & 55 & 46 & $0.00 \%$ & $0.00 \%$ & 0.6 \\
\hline Lombardia & 468 & 1488 & 1423 & $0.16 \%$ & $-0.75 \%$ & 17.0 \\
\hline Veneto & 3817 & 6916 & 5602 & $4.23 \%$ & $-0.96 \%$ & 79.2 \\
\hline Friuli-Venezia Giulia & 286 & 1528 & 945 & $0.13 \%$ & $-0.38 \%$ & 17.5 \\
\hline Liguria & 124 & 257 & 493 & $0.01 \%$ & $-0.04 \%$ & 2.9 \\
\hline Emilia-Romagna & 336 & 940 & 640 & $0.07 \%$ & $-0.74 \%$ & 10.8 \\
\hline Toscana & 3392 & 6099 & 4332 & $3.20 \%$ & $-1.01 \%$ & 69.8 \\
\hline Umbria & 73 & 248 & 173 & $0.05 \%$ & $-0.13 \%$ & 2.8 \\
\hline Marche & 1359 & 2243 & 1541 & $0.79 \%$ & $-0.21 \%$ & 25.7 \\
\hline Lazio & 432 & 925 & 1455 & $0.13 \%$ & $-0.55 \%$ & 10.6 \\
\hline Abruzzo & 197 & 324 & 300 & $0.04 \%$ & $-0.36 \%$ & 3.7 \\
\hline Molise & 407 & 479 & 428 & $0.12 \%$ & $-0.01 \%$ & 5.5 \\
\hline Campania & 420 & 953 & 1212 & $0.13 \%$ & $-0.41 \%$ & 10.9 \\
\hline Puglia & 6854 & 7895 & 8016 & $3.04 \%$ & $-0.72 \%$ & 90.4 \\
\hline Basilicata & 61 & 78 & 104 & $0.02 \%$ & $-0.06 \%$ & 0.9 \\
\hline Calabria & 168 & 223 & 369 & $0.04 \%$ & $-0.12 \%$ & 2.6 \\
\hline Sicilia & 620 & 973 & 1764 & $0.43 \%$ & $-0.94 \%$ & 11.1 \\
\hline Sardegna & 221 & 394 & 668 & $0.05 \%$ & $-0.45 \%$ & 4.5 \\
\hline Bolzano & 125 & 446 & 241 & $0.01 \%$ & $-0.35 \%$ & 5.1 \\
\hline Trento & 268 & 899 & 482 & $0.04 \%$ & $-0.34 \%$ & 10.3 \\
\hline Italy total & 20346 & 35418 & 32100 & $12.85 \%$ & $-10.21 \%$ & 405.5 \\
\hline
\end{tabular}

Table 1: Results on vineyards suitable for controlled origin wine 


\begin{tabular}{|c|c|c|c|c|c|c|}
\hline Region & Farms & \# Records & Changes & Added Area & $\begin{array}{c}\text { Subtracted } \\
\text { Area }\end{array}$ & Time (sec.) \\
\hline Piemonte & 528 & 866 & 1961 & $0.05 \%$ & $-0.28 \%$ & 9.9 \\
\hline Valle d'Aosta & 168 & 254 & 173 & $0.01 \%$ & $-0.01 \%$ & 2.9 \\
\hline Lombardia & 2312 & 3761 & 3172 & $0.38 \%$ & $-0.25 \%$ & 43.1 \\
\hline Veneto & 1346 & 3811 & 2593 & $0.33 \%$ & $-1.05 \%$ & 43.7 \\
\hline Friuli-Venezia Giulia & 1489 & 2931 & 2223 & $0.19 \%$ & $-0.19 \%$ & 33.6 \\
\hline Liguria & 868 & 1465 & 1321 & $0.06 \%$ & $-0.04 \%$ & 16.8 \\
\hline Emilia-Romagna & 436 & 918 & 814 & $0.10 \%$ & $-0.62 \%$ & 10.5 \\
\hline Toscana & 4558 & 11481 & 6747 & $0.83 \%$ & $-4.88 \%$ & 131.6 \\
\hline Umbria & 2200 & 4924 & 2466 & $0.18 \%$ & $-0.17 \%$ & 56.4 \\
\hline Marche & 3005 & 5850 & 3472 & $0.33 \%$ & $-0.09 \%$ & 67.0 \\
\hline Lazio & 4333 & 7363 & 7768 & $0.43 \%$ & $-0.80 \%$ & 84.4 \\
\hline Abruzzo & 5392 & 10664 & 5673 & $0.71 \%$ & $-0.94 \%$ & 122.2 \\
\hline Molise & 1732 & 3392 & 1841 & $0.24 \%$ & $-0.05 \%$ & 38.9 \\
\hline Campania & 10904 & 18092 & 13728 & $0.92 \%$ & $-0.67 \%$ & 207.3 \\
\hline Puglia & 10897 & 15251 & 13383 & $2.86 \%$ & $-1.48 \%$ & 174.8 \\
\hline Basilicata & 370 & 486 & 659 & $0.04 \%$ & $-0.15 \%$ & 5.6 \\
\hline Calabria & 2455 & 3391 & 4513 & $0.41 \%$ & $-0.89 \%$ & 38.9 \\
\hline Sicilia & 4224 & 7497 & 9630 & $2.00 \%$ & $-3.54 \%$ & 85.9 \\
\hline Sardegna & 642 & 1385 & 2528 & $0.07 \%$ & $-0.72 \%$ & 15.9 \\
\hline Bolzano & 11 & 21 & 19 & $0.00 \%$ & $-0.01 \%$ & 0.2 \\
\hline Trento & 1936 & 2400 & 1977 & $0.13 \%$ & $-0.05 \%$ & 27.5 \\
\hline Italy total & 59806 & 106203 & 86661 & $10.25 \%$ & $-16.87 \%$ & 1217.0 \\
\hline
\end{tabular}

Table 2: Results on vineyards not suitable for controlled origin wine

As for the first aspect, data obtained by the procedure were able to satisfy the balancing conditions in the totality of the cases $(100 \%)$. As for the second aspect, a positive feature for a general information reconstruction procedure is satisfying the requirements while not changing the data exceedingly. In the analyzed cases, in addition to the theoretical guarantee that the number of changes is minimal, we observe that the amount of the variations is always a small percentage. This means that the procedure was able to reconstruct information without distorting the data.

\begin{tabular}{|c|c|c|c|c|c|c|}
\hline Region & Farms & \# Records & Changes & Added Area & $\begin{array}{c}\text { Subtracted } \\
\text { Area }\end{array}$ & Time (sec.) \\
\hline Piemonte & 624 & 1682 & 1191 & $0.037 \%$ & $-0.016 \%$ & 19.2 \\
\hline Valle d'Aosta & 84 & 221 & 157 & $0.000 \%$ & $0.000 \%$ & 2.5 \\
\hline Lombardia & 1436 & 3784 & 2162 & $0.030 \%$ & $-0.069 \%$ & 43.3 \\
\hline Veneto & 5715 & 13604 & 11033 & $0.383 \%$ & $-0.670 \%$ & 155.8 \\
\hline Friuli-Venezia Giulia & 734 & 1955 & 1123 & $0.010 \%$ & $-0.002 \%$ & 22.4 \\
\hline Liguria & 264 & 645 & 458 & $0.001 \%$ & $0.000 \%$ & 7.3 \\
\hline Emilia-Romagna & 252 & 742 & 976 & $0.168 \%$ & $-0.042 \%$ & 8.5 \\
\hline Toscana & 3006 & 6571 & 6274 & $0.486 \%$ & $-0.319 \%$ & 75.2 \\
\hline Umbria & 513 & 1160 & 931 & $0.007 \%$ & $-0.003 \%$ & 13.2 \\
\hline Marche & 2210 & 5347 & 4615 & $0.229 \%$ & $-0.088 \%$ & 61.2 \\
\hline Lazio & 912 & 2023 & 1553 & $0.009 \%$ & $-0.002 \%$ & 23.1 \\
\hline Abruzzo & 1960 & 4540 & 2650 & $0.038 \%$ & $-0.103 \%$ & 52.0 \\
\hline Molise & 2006 & 4649 & 3817 & $0.047 \%$ & $-0.035 \%$ & 53.2 \\
\hline Campania & 2854 & 6538 & 4403 & $0.015 \%$ & $-0.003 \%$ & 74.9 \\
\hline Puglia & 18205 & 41924 & 34881 & $0.561 \%$ & $-0.527 \%$ & 480.3 \\
\hline Basilicata & 433 & 996 & 710 & $0.011 \%$ & $-0.005 \%$ & 11.4 \\
\hline Calabria & 506 & 1166 & 851 & $0.007 \%$ & $-0.008 \%$ & 13.3 \\
\hline Sicilia & 1117 & 2546 & 1772 & $0.035 \%$ & $-0.002 \%$ & 29.1 \\
\hline Sardegna & 223 & 512 & 407 & $0.006 \%$ & $-0.004 \%$ & 5.8 \\
\hline Bolzano & 23 & 96 & 83 & $0.012 \%$ & $-0.037 \%$ & 1.1 \\
\hline Trento & 889 & 2280 & 1344 & $0.005 \%$ & $-0.003 \%$ & 26.1 \\
\hline Italy total & 43966 & 102981 & 81391 & $2.098 \%$ & $-1.938 \%$ & 1180.0 \\
\hline
\end{tabular}

Table 3: Results on other cultivations 


\begin{tabular}{|c|c|c|c|c|c|c|}
\hline Region & Farms & \# Records & Changes & Added Heads & $\begin{array}{c}\text { Subtracted } \\
\text { Heads }\end{array}$ & Time (sec.) \\
\hline Piemonte & 704 & 947 & 719 & $0.317 \%$ & $-0.107 \%$ & 9.7 \\
\hline Valle d'Aosta & 38 & 51 & 38 & $0.000 \%$ & $0.000 \%$ & 0.5 \\
\hline Lombardia & 528 & 784 & 574 & $0.390 \%$ & $-0.130 \%$ & 8.0 \\
\hline Veneto & 3797 & 4449 & 4489 & $7.348 \%$ & $-2.763 \%$ & 45.4 \\
\hline Friuli-Venezia Giulia & 164 & 210 & 169 & $0.017 \%$ & $-0.285 \%$ & 2.1 \\
\hline Liguria & 71 & 92 & 71 & $0.000 \%$ & $0.000 \%$ & 0.9 \\
\hline Emilia-Romagna & 333 & 593 & 500 & $5.585 \%$ & $-8.403 \%$ & 6.1 \\
\hline Toscana & 1554 & 1878 & 1973 & $0.265 \%$ & $-0.037 \%$ & 19.2 \\
\hline Umbria & 124 & 160 & 124 & $0.000 \%$ & $-0.554 \%$ & 1.6 \\
\hline Marche & 1171 & 1488 & 1592 & $0.823 \%$ & $-0.428 \%$ & 15.2 \\
\hline Lazio & 302 & 380 & 305 & $0.000 \%$ & $0.000 \%$ & 3.9 \\
\hline Abruzzo & 215 & 286 & 217 & $1.634 \%$ & $0.000 \%$ & 2.9 \\
\hline Molise & 1003 & 1303 & 1281 & $0.437 \%$ & $-0.477 \%$ & 13.3 \\
\hline Campania & 387 & 471 & 394 & $0.001 \%$ & $0.000 \%$ & 4.8 \\
\hline Puglia & 3734 & 4144 & 4556 & $0.595 \%$ & $-0.570 \%$ & 42.3 \\
\hline Basilicata & 123 & 173 & 126 & $0.001 \%$ & $0.000 \%$ & 1.8 \\
\hline Calabria & 266 & 320 & 270 & $0.000 \%$ & $0.000 \%$ & 3.3 \\
\hline Sicilia & 338 & 441 & 340 & $0.000 \%$ & $0.000 \%$ & 4.5 \\
\hline Sardegna & 276 & 489 & 281 & $0.004 \%$ & $0.000 \%$ & 5.0 \\
\hline Bolzano & 127 & 181 & 127 & $0.000 \%$ & $0.000 \%$ & 1.8 \\
\hline Trento & 97 & 111 & 97 & $0.000 \%$ & $0.000 \%$ & 1.1 \\
\hline Italy total & 15352 & 18951 & 18243 & $17.418 \%$ & $-13.754 \%$ & 193.5 \\
\hline
\end{tabular}

Table 4: Results on livestock

The accuracy of the reconstructed information has been further evaluated by setting up a specific experiment. A large dataset of 274687 records representing all vineyards obtained from about 126000 farms, all exact, were perturbed by introducing random errors with uniform distribution at 3 different intensities, so that respectively about $1 \%, 5 \%$ and $10 \%$ of the microdata values have been changed. This was performed 20 times, in order to obtain statistically significant results, so 60 different large erroneous datasets were obtained. After this, the reconstruction procedure was applied to all of them, and the 60 obtained (corrected) data sets were compared to the original exact one.

Statistical indicators commonly used for measuring the differences between real and predicted values, such as the Relative Root Mean Square Error (RRMSR), are practically $0\left(<10^{-5}\right)$ for all the corrected datasets. This means that the quality of the reconstruction is fully satisfactory. However, in order to obtain more insight, we analyzed the reconstruction at an even greater detail: we compared each single reconstructed value to its original value, and checked whether it was exactly identical or not. Note that such test is extremely strict, probably beyond the requirements of a similar reconstruction process. The results are presented in Table 5. The percentage of reconstructed values that are exactly equal to the original values has been computed by subdividing the datasets on the basis of the number of errors actually introduced in each farm. Clearly, those percentages lower when the number of errors introduced in the farm increases, but accuracy is anyway extremely high. Even when the farm data contain a considerable number of errors (from 4 to 10 , that is often more than what happens in usual practice), the reconstructed values are exactly equal to the original ones in a very high percentage of the cases.

\begin{tabular}{|c|r|r|r|}
\hline \multirow{2}{*}{$\begin{array}{c}\text { Errors per } \\
\text { Farm }\end{array}$} & \multicolumn{3}{|c|}{ Percentage of Exactly Reconstructed Values } \\
\cline { 2 - 4 } & $\begin{array}{c}\text { Perturbation at } \\
\mathbf{1 \%}\end{array}$ & $\begin{array}{c}\text { Perturbation at } \\
\mathbf{5 \%}\end{array}$ & $\begin{array}{c}\text { Perturbation at } \\
\mathbf{1 0 \%}\end{array}$ \\
\hline 1 & $99.9 \%$ & $99.9 \%$ & $99.9 \%$ \\
\hline 2 & $98.1 \%$ & $98.2 \%$ & $98.2 \%$ \\
\hline 3 & $86.0 \%$ & $86.6 \%$ & $83.4 \%$ \\
\hline $4 \div 10$ & $81.8 \%$ & $56.3 \%$ & $49.1 \%$ \\
\hline
\end{tabular}

Table 5: Accuracy of the reconstruction process 


\section{Conclusions}

Information Reconstruction is a crucial task in the case of large surveys, such as a Census of Agriculture, as well as for other applications of database processing. A typical problem arising in the described Census consists in checking, and correcting when needed, the areas declared by each farm for each cultivation. This type of balancing problem is extremely important and has a great economical relevance. Moreover, in contexts different from the Census, the possibility of reconstructing exact values could be useful for counteracting opportunistic behaviors, e.g. willingly erroneous declarations for influencing resources allocation or production plans.

Similar problems could be formulated in different manners. This particular Census problem has very specific aims and requirements, and it was deemed that they were better represented by the proposed mixed integer linear model (11). The procedure has been tested in the case of the Italian Census of Agriculture 2010 with specific respect to the 4 most important cases. Clearly, the proposed class of models is not limited to the case of an Agricultural Census, but can be used for other problems sharing the same characteristics, in particular the presence of balance requirements and minimum change objective. Results are very encouraging both from the computational and from the data quality point of view. The sequence of arisen mixed integer problems can be solved to optimality by using a state-of-the-art implementation of branch-and-cut procedures. Each single model is solved in extremely short times. In the totality of the cases the reconstructed information was able to satisfy the balancing conditions without excessively distorting the data, as resulted from the analysis of the variations introduced in the whole datasets. Moreover, a specific experiment proves that the reconstructed information was exactly equal to the original uncorrupted one in an exceedingly high percentage of the cases.

\section{References}

1. Bacharach, M.: Matrix rounding problems. Management Science 12(9), 732-742 (1966)

2. Bacharach, M.: Biproportional Matrices and Input-Output Change. Cambridge University Press, Cambridge, UK (1970)

3. Banff Support Team: Functional Description of the Banff System for Edit and Imputation System. Quality Assurance and Generalized Systems Section Tech. Rep. Statistics Canada (2003)

4. Bankier, M.: Canadian Census Minimum change Donor imputation methodology. In Proceedings of the Workshop on Data Editing, UN/ECE, Cardiff, United Kingdom (2000).

5. Bertsimas, D. and Tsitsiklis, J.N.: Introduction to Linear Optimization. Athena Scientific, Belmont, Massachusetts (1997)

6. Bomze, I.M., Locatelli, M.: Separable standard quadratic optimization problems. Optimization Letters 6(5), 857-866 (2012)

7. Bourbaki, N.: Topological vector spaces. Springer-Verlag, Berlin, Germany (1987)

8. Boyd, S. and Vandenberghe, L.: Convex Optimization. Cambridge University Press, Cambridge, UK (2004)

9. Bruni, R.: Discrete Models for Data Imputation. Discrete Applied Mathematics 144(1), 59-69 (2004)

10. Bruni, R.: Error Correction for Massive Data Sets. Optimization Methods and Software 20(2-3), 295-314 (2005)

11. Bruni, R. and Bianchi, G.: A Formal Procedure for Finding Contradictions into a Set of Rules. Applied Mathematical Sciences 6(126), 6253-6271 (2012)

12. De Waal, T.: Computational Results with Various Error Localization Algorithms. UNECE Statistical Data Editing Work Session, Madrid, Spain (2003)

13. De Waal, T., Pannekoek, J., Scholtus, S.: Handbook of Statistical Data Editing and Imputation. Wiley Handbooks in Survey Methodology, John Wiley \& Sons, Inc.: New York, NY (2011)

14. European Council Regulation (EEC) No $357 / 79$ of 5 February 1979 on statistical surveys, EEC Documentation (1979)

15. Fellegi, I.P. and Holt, D.: A systematic approach to automatic edit and imputation. Journal of the American Statistical Association 71, 17-35 (1976)

16. Fuller, W.A.: Measurement Error Models. Wiley Series in Probability and Statistics, John Wiley \& Sons, Inc.: New York, NY (2006)

17. Garey, M.R. and Johnson, D.S.: Computers and Intractability: A Guide to the Theory of NPCompleteness. W.H. Freeman and Co, San Francisco, CA (1979)

18. Hastie, T., Tibshirani, R., Friedman, J.: The Elements of Statistical Learning: Data Mining, Inference and Prediction, Springer, New York, NY (2001)

19. Hochbaum, D.S. and Shanthikumar, J.G.: Convex separable optimization is not much harder than linear optimization. Journal of the ACM 37(4), 843-862 (1990)

20. IBM: Ilog Concert Technology 12.1 Reference Manual. International Business Machines Corporation (2009)

21. IBM: Ilog Cplex 12.1 Reference Manual. International Business Machines Corporation (2009) 
22. Kalantari, B., Lari, I., Ricca, F., Simeone, B.: On the complexity of general matrix scaling and entropy minimization via the RAS algorithm. Mathematical Programming, Ser. A 112, 371-401 (2008)

23. Klösgen, W. and Żytkow, J.M. (Eds.): Handbook of Data Mining and Knowledge Discovery, Oxford University Press: Oxford, UK (2002)

24. Lyberg L.E., Biemer P., Collins M., De Leeuw E.D., Dippo C., Schwarz N., Trewin D. (Eds.): Survey Measurement and Process Quality, Section C, post survey processing and operations. John Wiley \& Sons, Inc.: New York, NY (1997)

25. Mucherino, A., Papajorgji, P., Pardalos, P.M.: Data Mining in Agriculture, Springer: New York, NY (2009)

26. Nemhauser, G.L. and Wolsey, L.A.: Integer and Combinatorial Optimization. John Wiley \& Sons, Inc.: New York, NY (1999)

27. Riera-Ledesma, J. and Salazar-Gonzalez, J.J.: New Algorithms for the Editing and Imputation Problem. UNECE Statistical Data Editing Work Session, Madrid, Spain (2003)

28. Ramakrishnan, R. and Gehrke, J.: Database Management Systems ( $3^{\text {rd }}$ edition). McGraw-Hill: New York, NY (2003)

29. Schneider, M.H. and Zenios, S.A.: A comparative study of algorithms for matrix balancing. Operations Research, 38(3), 439-455 (1990)

30. Schrijver, A.: Combinatorial Optimization. Springer, Berlin; New York (2003)

31. Winkler, W.E.: State of Statistical Data Editing and current Research Problems. In Proceedings of the Workshop on Data Editing, UN/ECE, Rome, Italy (1999) 